CLAWAR 2018: 21st International Conference on Climbing and Walking Robots and the Support Technologies for Mobile Machines, Panama City, Panama, 10-12 September 2018

\title{
MINIATURE MAGNETIC ROBOTS FOR IN-PIPE LOCOMOTION
}

\author{
GEORGE H MILLS ${ }^{\dagger}$ \\ Department of Mechanical Engineering, University of Leeds, \\ Leeds, LS2 9JT, United Kingdom
DR. JASON H W LIU, DR. BILAL Y KADDOUH, DR. ANDREW E JACKSON, PROF. ROBERT C RICHARDSON
Institute of Design Robotics and Optimisation, Lab G.54, Leeds, LS2 9JT, United Kingdom

\begin{abstract}
Inspection of both small and large diameter bore pipelines for pipe integrity and defect identification with a single system has previously been impractical; especially using wall-press locomotion methods with low adaptive range. A miniature magnetic wallclimbing robot has been developed as a robotic solution for the inspection of $50 \mathrm{~mm}$ bore diameter pipelines which can scale in-pipe geometry obstacles to access larger connected pipelines. Using magnetic arrays directed through steel flux plates within the wheels, the robot uses magnetic forces to adhere to the pipe. The system is 3D printed and includes soft printed material rubber wheels. The robot prototype is wirelessly driven, controlled remotely through serial Bluetooth communication radio at $2.4 \mathrm{GHz}$ rated up to $100 \mathrm{~m}$. The robot's unique compact geometry and magnetic design allows it to scale concave rightangle wall cases in just a 50mm diameter bore. By entering pipe networks through these small existing access points the robot removes the need for expensive drilling procedures required to fit launch vessels.
\end{abstract}

Index Terms - In-Pipe Robot; 3D Printed Robot; Magnetic Robot; Pipe Inspection

\section{Introduction}

The field of in-pipe robotics is rapidly growing as pipeline networks around the world begin to reach the end of their initial design lives. Maintenance and repair of these networks can be costly and damaging to the environment due to the unnecessary excavation of healthy pipelines. Inspection robots can be used to investigate sections of pipe flagged for replacement and can

This work was supported by National Grid Gas Transmission (NGGT) and its project partners Synthotech, Premtech, and Pipeline Integrity Engineers as part of Project GRAID: Gas Robotic Agile Inspection Device. ESPRC Grant Number: 1657711 
determine the true interior condition. Types of in-pipe robotic locomotion can be categorized using basic elements, the elements presented in Figure 1 expand on the types of locomotion discussed in previous in-pipe hybrid literature reviews [1]. Modern in-pipe robots combine basic locomotion elements to form hybrid in-pipe systems with the ability to traverse a wide range of pipe obstacles and diameters [2]. The simplest of these being wheeled and caterpillar type robots (B \& D). Specialized in-pipe methods of movement include, screwing, wall-press, and inchworm systems (C, E, \& F). These locomotion methods are often combined with the more advanced methods, snake, walking, and magnetic ( $\mathrm{G}, \mathrm{H}, \& \mathrm{~J})$. The most widely used hybrid is wheeled wall-press systems due to their ability to use the pipe walls for traction. Adaptable Quad Arm Mechanism (AQAM) is a hybrid wheeled wall-pressing robot for $260 \mathrm{~mm}-300 \mathrm{~mm}$ pipes, consisting of four arm mounted wheels in a single plane. The robot has impressive maneuverability due to its four independently controllable arms and swivel mechanism [3]. Multifunction Robot for INSPECTion of pipeline (MRINSPECT) is a wheeled wall-press hybrid in-pipe robot series designed at Sungkyunkwan University. The robot can perform all types of in-pipe geometry problems shown in Figure 2. MRINSPECT uses a multi-axial differential gear system to control each of its four wheeled legs angles through active bevel drive connections [4].

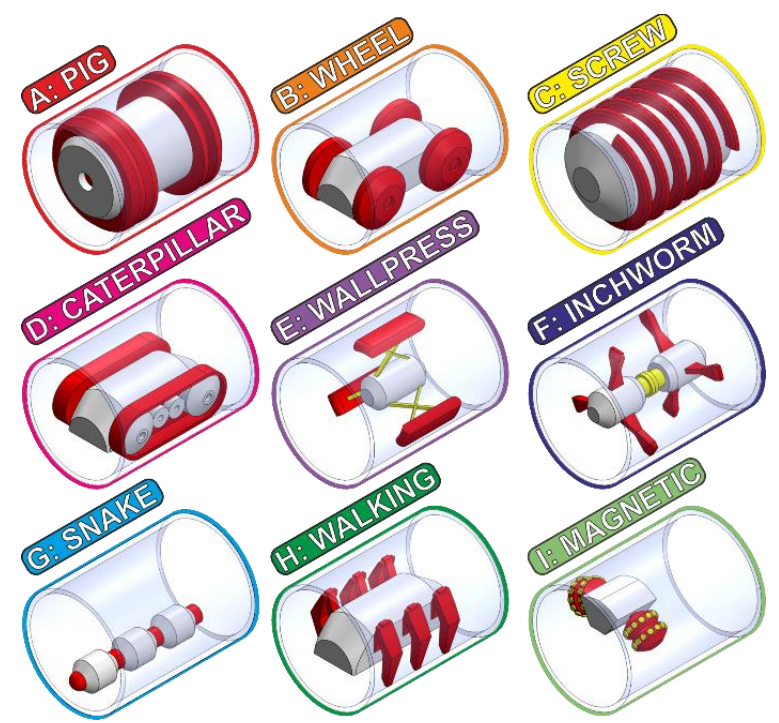

Figure 1: Basic in-pipe robotic locomotion types A - I. 
Hanyang University developed a single-plane wheeled system. The problem faced with single plane contact is stability, any loss of wall contact in these designs will de-centralize the robot and make recovery extremely difficult [5]. Heli-Pipe is a series of wall-press systems that have a diametric adaptive ability of $10 \mathrm{~mm}$, as a result four different prototypes were made, ranging from $170 \mathrm{~mm}$ to $40 \mathrm{~mm}$ [6]. Caterpillar wall-press robots are also popular as a higher traction alternative to wheeled hybrid systems. Kanagawa University developed a hybrid of caterpillar and wall-press components, built from modular units each containing a driving caterpillar track. Connecting three or more units allows the robot to drive in-pipe, should a larger diameter need to be traversed the number of driving units can be increased [7]. Pipe Adaptive Robot of YonSei University (PAROYS-II) uses an actively controlled pantograph mechanism with a partially passive spring mechanism, this allows large changes to be controlled and small obstacles to be ignored. Its use of a second set of articulated caterpillar tracks allows a huge adaptive range of $400 \mathrm{~mm}-700 \mathrm{~mm}$ [6]. SPRING is a screw type wall-press robot developed at Osaka University, although it relies on full wall traction it is unlike traditional full-bore wall-press systems which keep their chassis centralized in the pipe [9]. Snake robots are popular in industry pipe applications, they maximize space available and can be easily made to be modular in design allowing them to be flexibly suited to different tasks. PIRATE snake-like, and modular in nature featuring articulated clamping modules that can actively change the height of the robot to adapt to changes in pipe diameter [10]. The PipeTron series developed by HiBot, Tokyo is a multitude of robotic in-pipe exploration snake robots. Predominantly designed for tight bend systems such as refineries and chemical plants, the system is tethered for instant retrieval and consists of passively articulated wheels connected in a series [11]. The Explorer series is a multitude of industrial snake robots designed for the inspection of live gas networks under operating conditions, however it requires a full bore to operate with little adaptability ranging from just $150 \mathrm{~mm}-200 \mathrm{~mm}$ [12]. 


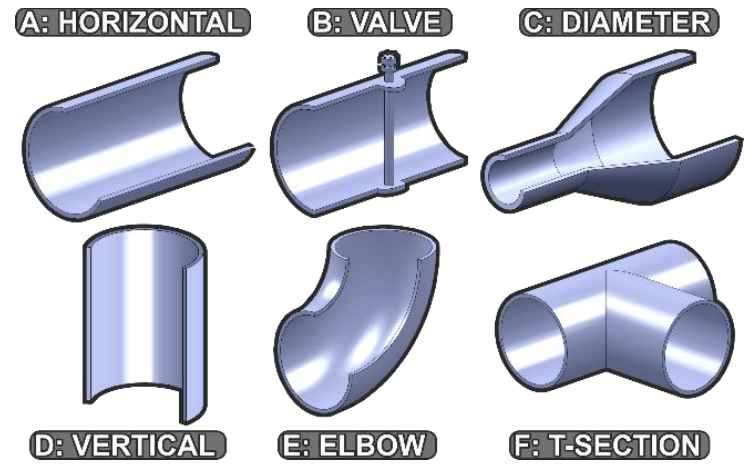

Figure 2: Common in-pipe obstacles A-F.

In ferrous pipelines, magnetic systems have all the locomotive advantages of wallpress without the need for adaptive diameter mechanisms. The first magnetic inpipe robot was developed by the Osaka Gas Company in 1995, the system was a dual wheeled magnetic concept for the inspection $150 \mathrm{~mm}-600 \mathrm{~mm}$ iron pipelines [13]. An advanced example of a magnetic wheeled in-pipe robot would be MagneBike. It can steer in a large range of in-pipe diameters, and can make obstacles such as T-Sections become trivial [14]. Synthotech [15] is making innovative progress towards complete pipe inspection with two robotic platforms targeting different challenges. The first; the Tier One Replacement System (TORS), a snake pipe robot focusing on lower diameter consumer pipeline replacement. Project Gas, Robotic Agile Inspection Device (GRAID) [16], will inspect the National Grid Gas Network under live operating conditions using a magnetically adhered caterpillar system. Magnetic robots are also used in out-pipe cases to traverse walls, ship hulls, and ferrous structures such as the boiler tube inspection prototype robot [17]. Omni-directional wheels used in conjunction with magnetics can lead to extremely maneuverable out-pipe robots such as Omni-Climber [18].

\section{In-pipe robot design}

The proposed in-pipe robot is intended to travel from small diameter pipelines to larger bores. The target inspection network consists of ferrous pipelines in the diameter range of $50 \mathrm{~mm}-1250 \mathrm{~mm}$. Magnetic adhesion is a suitable method in this case, performing the function of adhesion without the need for a full-bore wall-pressing system. As seen in other wall-pressing designs a full-bore adaption mechanism severely limits the range of pipelines that can be inspected by a single robot, even in the best case: the robot called PAROYS-II has a full adaption range 
of $300 \mathrm{~mm}$ [8]. The proposed robot will be specified to enter a $50 \mathrm{~mm}$ inner diameter pipe and be able to overcome obstacles present in the network. For miniature wall-climbing robots, obstacles in Figure 2 can be simplified to distinct cases shown in Figure 3 in which a bend is either concave, or convex. Completion of both convex and concave geometries using magnetic systems is challenging without the use of articulated systems or many actuators allowing multiple degrees of freedom. Driving directly up to a convex case with magnetic wheels causes them to lock in place as the magnets act with equal force to each wall in contact.

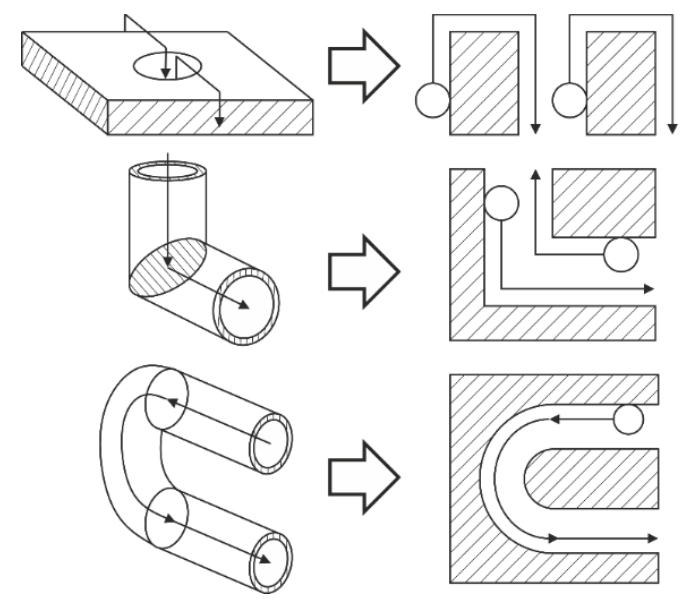

Figure 3: Simplified in-pipe geometry.

The concave case is equally challenging, as the wheels reach the right angle, magnetic force drops dramatically as the total potential magnetic flux transferred into the steel decreases causing slip at the wheels. The design challenge is to overcome these types of obstacle using a robot that stays within the tight space constraints of a $50 \mathrm{~mm}$ pipeline. For this to be possible the robot profile must be below the given diameter with a clearance of the magnetic wheels such that they do not attract or lock with the top side of the inner pipe.

\subsection{Robot Design Overview}

The magnetic pipe robot, shown in Figure 5 was designed to enter $50 \mathrm{~mm}$ internal diameter ferrous pipelines. The robot is equipped with magnetic wheels, a wireless control system, and two motors, it is $3 \mathrm{D}$ printed, including soft rubber wheels printed in-situ using soft printed material. Many basic vehicle designs were considered to save space within the pipe. The planar wheel (bike like) 
configuration as used in MagneBike [14] Figure 4 (a), allows for efficient placement of magnetic wheels however it requires stabilisers to remain laterally stable (b). In a 50mm pipe, space is paramount and so a two-wheel vehicle (d) has been designed which grants efficient motor placement along the length of the pipe, this reduces the length and complexity vs both a 4 wheeled magnetic vehicle (c), and caterpillar system (e).

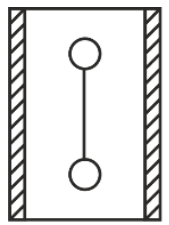

(a)

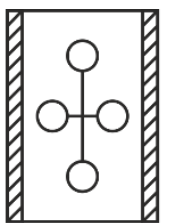

(b)

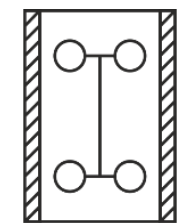

(c)

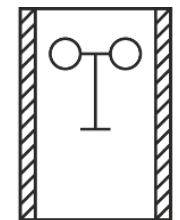

(d)

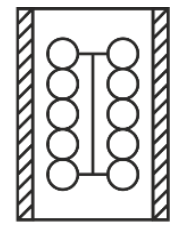

(e)

Figure 4: Magnetic robot skeleton base designs $(\mathrm{a}-\mathrm{e})$.
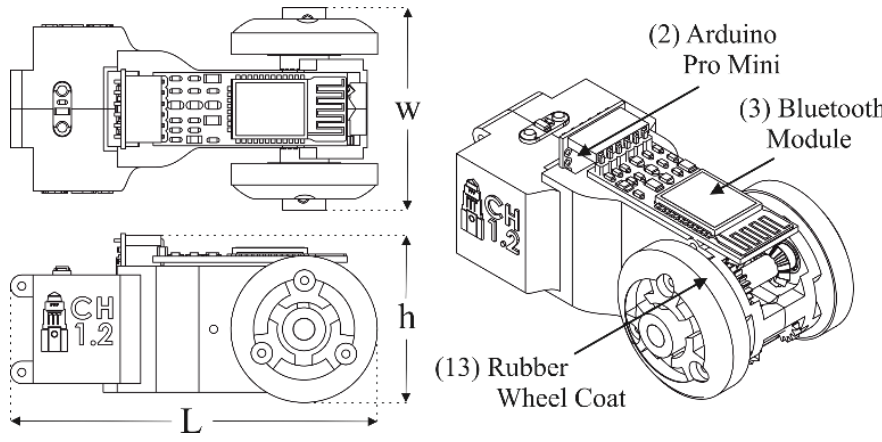

Figure 5: (a) Geometry of the magnetic robot.

$L \times w \times h=85.10 \mathrm{~mm} \times 46.88 \mathrm{~mm} \times 38.65 \mathrm{~mm}$

The main body of the robot is printed as two separate halves and screwed together after the addition of motors, electronics and battery. The wireless Bluetooth radio transceiver is mounted on top of the chassis directly with the Arduino FTDI. This allows reprogramming later by detaching the radio.

\subsection{Transmission System}

Due to the size constraints when designing a robot for a $50 \mathrm{~mm}$ diameter pipe, the motors are mounted perpendicular to driveshaft and the wheels. The motors sit parallel one on top of the other, each drive one gear train with transmission through a bevel gear, shown in Figure 6. The driveshaft then transmits power through the spur gear on the opposite side to drive the wheel with a transmission 
ratio of 1.2:1. These sections are 3D printed, they are assembled with 5mm I.D. $8 \mathrm{~mm}$ O.D. bearings which are push fit into the chassis during assembly. These allow smooth rotation and locating the centers for transmission assembly, keeping meshing distance of the gears constant. Although the motors are interchangeable depending on the desired gear ratio the current 250:1 motors allow a max wheel speed of 80 RPM.

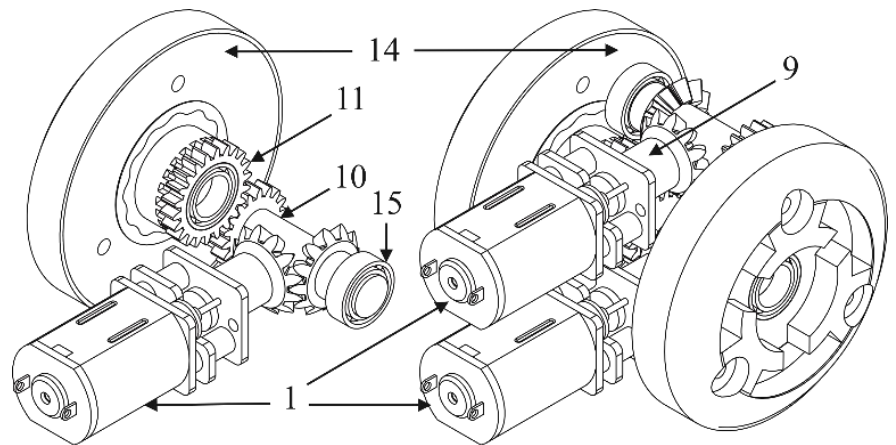

(a) Single Wheel Transmission (b) Whole Drive Assembly

Figure 6: Transmission system, \& Motor Layout, with part No. 1: Motor, 9: Gear, 10: Driving Shaft, 11: Wheel Gear, 14: Magnetic Wheels.

The prototype was formed using Objet material VeroWhite $(60 \mathrm{~g})$ and Tango+ $(4 \mathrm{~g})$ as well as breakaway support $(186 \mathrm{~g})$ to form a complete model. The total build time for one robot is $2 \mathrm{hrs}: 45 \mathrm{~min}$ and requires an hour of support cleaning from surrounding material due to the delicacy and small size of the robot parts. Shown in the Table 1 is the bill of materials required to assemble the robot. The price is quoted as $£ 171$ to produce one unit, however the printing costs for the chassis account for $66 \%$ of this price. This price is based on the production of one robot, charged at a $£ 25$ hourly rate for use of the Objet 1000 printer and a printing time of 3 hours. These costs could be reduced if the robot were to be printed in batches.

\subsection{Wireless Driving System}

The robot is controlled using a 3.3V version of the Arduino Pro Mini which interfaces wirelessly over serial using the SparkFun Bluetooth Mate Silver. The $3.3 \mathrm{~V}$ level logic voltage uses less power than $5 \mathrm{~V}$ version of the pro mini at the cost of processing speed $(8 \mathrm{MHz}$ vs $16 \mathrm{MHz})$ however it is directly compatible with all secondary components and does not require a voltage regulator. Bluetooth radio was chosen for the same reason, when idle it draws much less current than 
Wi-Fi options such as the ESP8266 (50mA vs 170mA during communication). As this robot is designed to operate in $50 \mathrm{~mm}-1250 \mathrm{~mm}$ diameter pipes, the Bluetooth $2.4 \mathrm{GHz}$ module will only be effectively operational in $80 \mathrm{~mm}$ $1250 \mathrm{~mm}$ diameter pipes. We are currently investigating the use of a $5.8 \mathrm{GHz}$ module for smaller diameter pipes $(50 \mathrm{~mm}-80 \mathrm{~mm})$ in future versions. For the purpose of this proof of concept, the Bluetooth module achieves the desired outcomes. A SparkFun dual motor driver breakout board is used to control direction of the two motors and hence steering, and a 3.3V LED is used as a power indicator. The whole system runs on a 260mah 7.4V Lithium Polymer (LiPo) rechargeable battery which supplies logic voltage to the Arduino, and raw voltage to the dual motor driver board. Commands are given to the Pro Mini via serial sent from a master computer running LabVIEW. The additional circuitry required increases assembly time in terms of the time it takes to solder, estimated at half an hour. The circuit boards and LED's simply slide into the printed grooves and are held in place by frictional forces. A summary of the electronic materials used in the production of one unit, including price and weight can be found in Table 1.

\subsection{Magnetic Wheel System}

The magnetic wheels were designed to increase the strength of the magnets by redirecting the flux of the magnets to focus it into the tracks. Instead of having magnets directly in contact with the inner surface of the pipe, they are housed between two steel plates, Figure 7. This has two benefits; redirection of the magnetic flux into the steel plates, and protection of the neodymium magnets. Corrosion and shock impact can cause degradation and fracturing of the magnets, by encapsulating them rather than placing them in direct contact with the pipe wall they are significantly safer. The magnets used in the wheel assembly are N42 $6 \times 4 \times 2 \mathrm{~mm}$ rectangular magnets with a pull strength of $0.75 \mathrm{~kg}$. These magnets have their north and south faces on opposite sides of the $6 \mathrm{~mm} \times 4 \mathrm{~mm}$ faces, which are in contact with the plates. The evenly spaced circular array of 9 magnets shown in Figure 7 is held within the wheel using a 3D printed spacing layer, this smooths out the flux dissipation to maintain an even distribution of tractive force as the wheel rotates. 


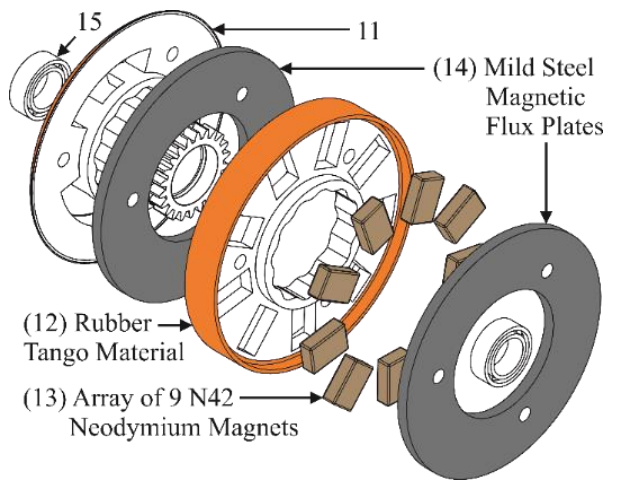

Figure 7: Exploded view of the $34 \mathrm{~mm}$ diameter wheel assembly.

The wheel is connected using three screws which locate the entire assembly ensuring the wheel lines up optimally as intended in the CAD model. The through holes are seen in Figure 7. The flux lines run from one side the plate back, through the $1.5 \mathrm{~mm}$ steel, into the pipe wall, and back through the second plate with the opposite face. By focusing the flux through the pipe wall in this way the magnetic absorption force increases, and a higher traction is generated at the wheel. Figure 8 presents a close up of a central wheel cross-section and shows the wheel in contact with a flat steel surface. The distance between the magnetic flux plates and the steel contact surface are seen to be offset by the rubber tango material. The distance between these two surfaces is $0.5 \mathrm{~mm}$ on a flat plate and up to $3.5 \mathrm{~mm}$ in a $50 \mathrm{~mm}$ diameter pipe section.

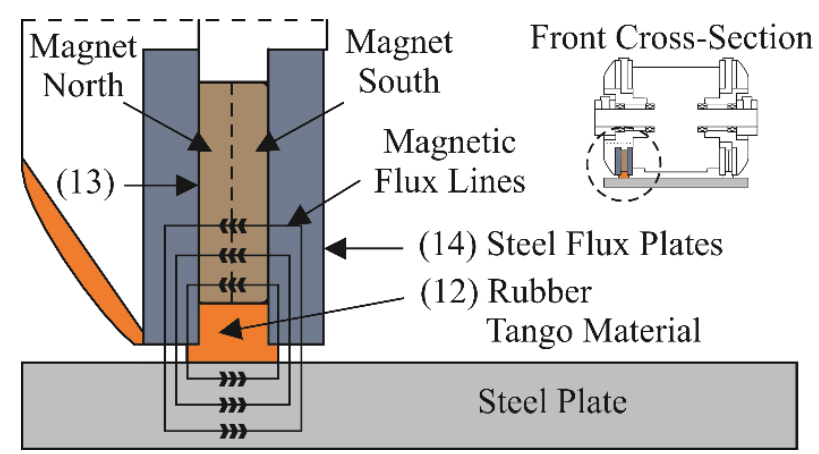

Figure 8: Magnetic wheel cross-section flux diagram. 


\section{Robot Performance}

The robot was printed, wheel flux plates laser cut, and assembled. The complete system was then analyzed in terms of locomotive capabilities, magnetic force, and maneuverability, the finished robot is shown in a $50 \mathrm{~mm}$ pipe Figure 9.

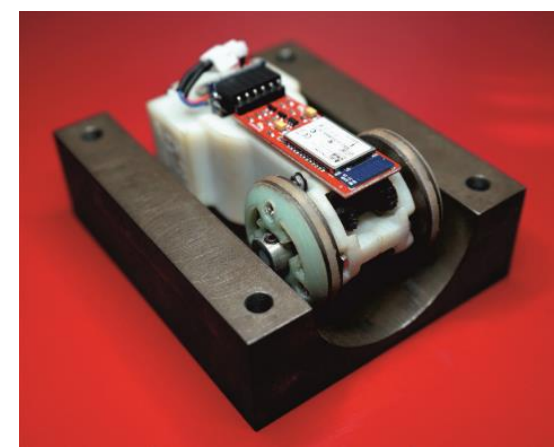

Figure 9: Assembled robot in a 50mm I.D. half-pipe.

The magnetic force generated by the wheels was tested on mild steel plates of varying diameter. The plates were fastened to a testing rig using $3 \mathrm{D}$ printed spacers, seen in orange, the robot was clamped to the 10 Newton load cell of the Emperor Force measurement device, Figure 10, and then pull force tests were undertaken. The tests followed the same method; initially the load cell was zeroed and the robot lowered down into contact with the plate until the load was at zero again. The robot was then pulled off the plate at a rate of $0.5 \mathrm{~mm} / \mathrm{sec}$ until a height was reached where the magnetic field no longer has influence $(30 \mathrm{~mm})$. The experiment was repeated 5 times for each plate size. The setup for this experiment shown in Figure 10, the results; Figure 11. It should be noted that the robot was first placed in a compression pre-loaded state to reach equilibrium with the magnetic force. This pre-loaded state is highlighted before each peak force point in Figure 11.

Figure 11 shows that the pull force case for flat plate produces significantly stronger force (almost triple) when compared to the $50 \mathrm{~mm}$ case. The uneven contact of the robot wheels in the $50 \mathrm{~mm}$ diameter pipe case results in sub-optimal transfer of magnetic flux through the steel. The inner flux plates are a minimum of $3 \mathrm{~mm}$ from the pipe walls making a complete flux flow through the two sides difficult. 


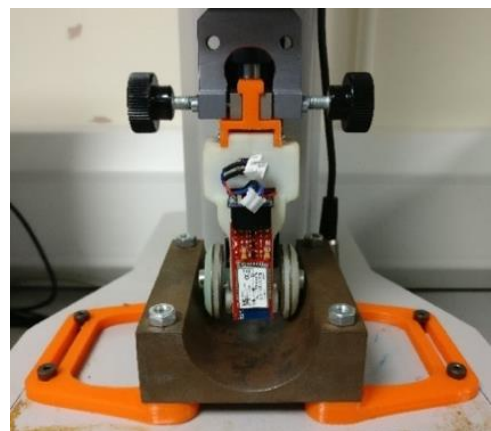

Figure 10: Single column linear force tester with a 10N load cell.
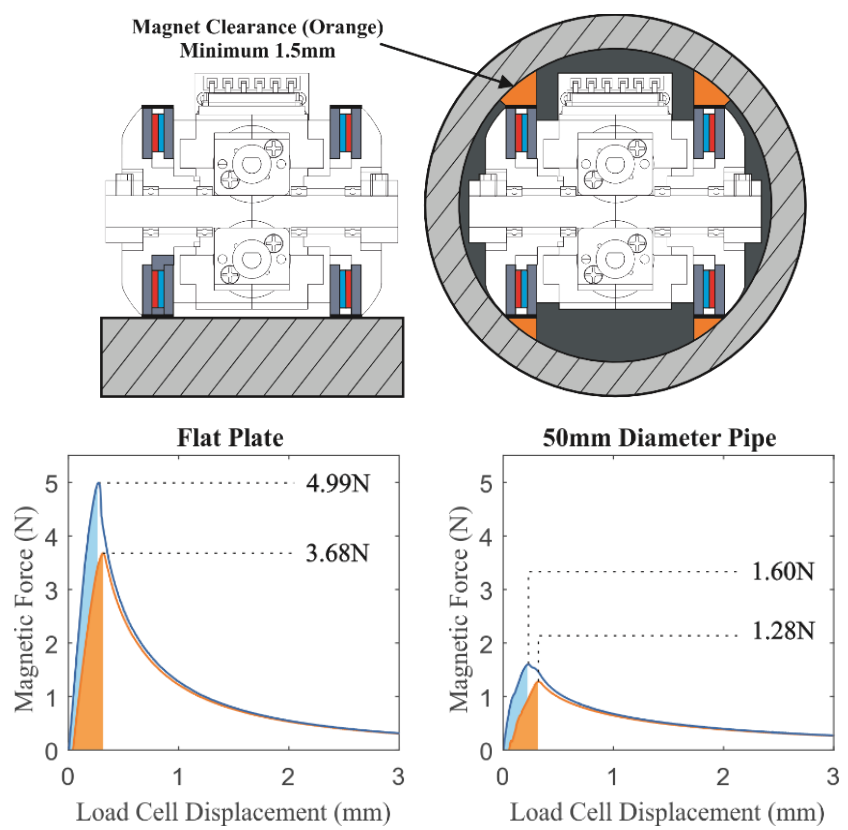

Figure 11: Magnetic pull force experiment result, pull force (blue), connection force (orange). In Figure 11, the peak pull force required to remove both wheel is highlighted in blue, whereas the re-attachment force is shown in orange. The shaded areas of the graphs indicate that the robot is in a compressed state.

Table 1: Standard deviation of 5 experiment samples.

\begin{tabular}{|l|c|c|c|c|}
\hline & $\begin{array}{l}\text { Flat Plate } \\
\text { Pull }\end{array}$ & $\begin{array}{l}\text { Flat Plate } \\
\text { Push }\end{array}$ & $\begin{array}{l}50 \mathrm{~mm} \\
\text { Pull }\end{array}$ & $\begin{array}{l}50 \mathrm{~mm} \\
\text { Push }\end{array}$ \\
\hline $\begin{array}{l}\text { Standard } \\
\text { Deviation }\end{array}$ & 0.017 & 0.002 & 0.114 & 0.009 \\
\hline
\end{tabular}


Magnetic wheels can encounter problems in cases where one wheel has more than one point in contact with a ferrous surface. The wheel can become stuck as to move in either direction it first has to overcome the unwanted magnetic force produced. The forces involved in this situation are described in Figure 12, where the robot is driving up a wall at a 90-degree concave angle. The robot is capable of completing the case presented in Figure 12 in a pipe, where traction is minimum. The robot has been designed with this worst case scenario in mind; travelling vertically in-pipe in the lowest diameter where magnetic force is minimum. This scenario results in lowest traction case, designing to complete this ensures the robot can perform any other section with a wider inner diameter or preferable orientations where magnetic or traction force applied is higher.

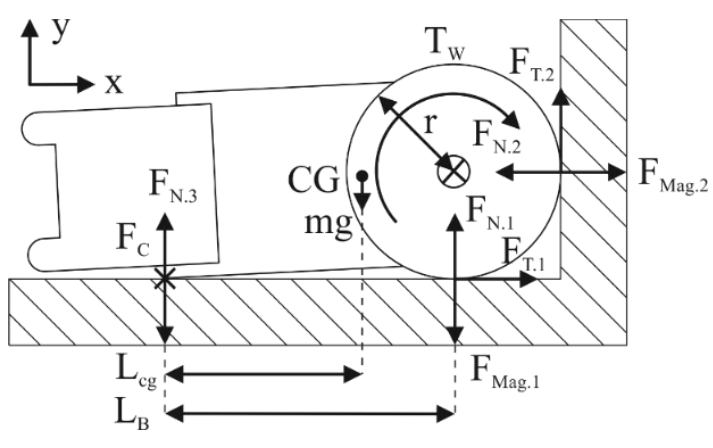

Figure 12: Forces acting at point of 90-degree wall contact.

For the wheel to drive up the wall from this position, the traction force $F_{T .2}$ must overcome the wheels magnetic pull $F_{\text {Mag.1. }}$. In this scenario the weight of the vehicle must also be overcome making this the most difficult case in terms of tractive effort. Balancing the forces around the point of contact and assuming the wheel is at the point just before accelerating up the wall, traction $F_{T .2}$ will equal $F_{\text {Mag.1 }}$ plus the weight component, equation (1).

$$
F_{T .2} *\left(L_{B}+r\right)_{c}=F_{\text {Mag. } 1} *\left(L_{B}\right)-m g\left(L_{B}-2 L_{c g}\right)
$$

The magnetic forces required to allow this robot to provide enough traction to overcome weight means that the mg component is comparatively low (2).

$$
-0.144 * 9.81 *(0.047-0.066)=0.0276 \mathrm{~N}
$$

Balancing forces in terms of $F_{T .2}$ the total force that the wheel traction must overcome to climb the wall can be determined by equations (3), and (4).

$$
\begin{gathered}
F_{T .2}=\frac{F_{\text {Mag.1 }} *\left(L_{B}\right)}{\left(L_{B}+r\right)}+0.0276 \\
F_{T .2}=2.89+0.0276=2.91 \mathrm{~N}
\end{gathered}
$$


To accelerate up the wall at this equilibrium point the traction force must satisfy the equation (5). Maximum tractive force that can be applied at $F_{\text {T.2.MAX }}$ is governed by equation (6):

$$
\begin{gathered}
F_{T .2}>\frac{F_{M a g .1^{*}\left(L_{B}\right)}}{\left(L_{B}+r\right)}+m g \sin (\varnothing) \\
F_{T .2 . M A X}=\mu_{S} F_{N .2}
\end{gathered}
$$

Where: $\mu_{s}=$ Static coefficient of friction of wheels

To satisfy the traction requirements, the static coefficient of friction, $\mu_{s}$ must be maximized. In the scenario outlined in Figure $12 F_{N .2}$ is equal to the magnetic force from Figure 11; 4.99N (lowest recorded force), and $F_{T .2}$ is equal to $2.91 \mathrm{~N}$ from equation (4). When entered into (7) the minimum required static friction coefficient can be obtained:

$$
\mu_{s}>\frac{F_{T .2}}{F_{N .2}} \quad \mu_{s}>0.58
$$

In Figure 13 the robot is shown driving on a steel floor directly up to a 90-degree steel wall in replica of the case outlined in Figure 12.

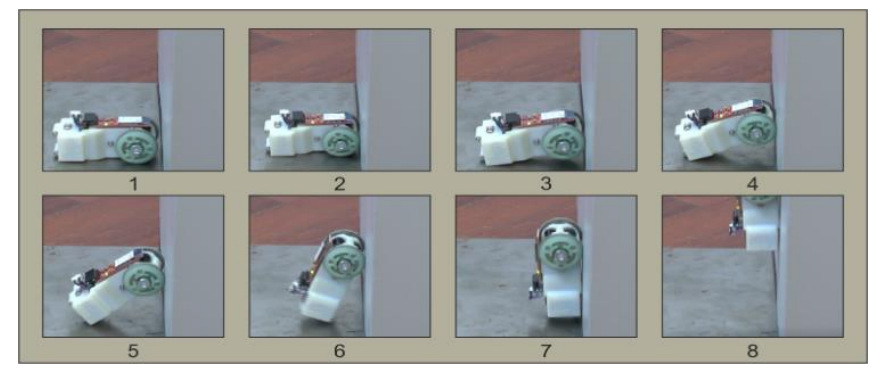

Figure 13: Magnetic robot climbing 90-degree from steel to steel.

The robot is optimized for high magnetic force on flat plate travel, this is due to the decreased risk in lower diameter pipelines. In large pipelines gas flow will likely be at the highest rate, hence a larger normal force will be required to maintain friction levels necessary to remove wheel slip. The robot can move at a top speed of $90 \mathrm{~mm} / \mathrm{sec}$ on a flat steel plate (Maximum magnetic attraction case) and can currently drive from any orientation. The robot is capable of completing a $180^{\circ}$ pipe bend with minimum radius of $25 \mathrm{~mm}$ within a $50 \mathrm{~mm}$ pipeline. Driving straight around the inside of a pipeline is possible as when the robot reaches wall angles greater than 90 degree's the chassis simply hangs upside-down. This version requires that you turn around at the top of the pipe as the Bluetooth mate module blocks wheel contact. Removing additional actuators to climb step cases greatly reduced the size of the robot however currently the robot is incapable of 
convex right-angle cases due to the limited step space between the wheel and the chassis; currently $2.4 \mathrm{~mm}$.

\section{Conclusion}

A wheeled-magnetic in-pipe robot has been designed to enter small $50 \mathrm{~mm}$ pipelines found within larger pipe networks. These small entrances of $50 \mathrm{~mm}$ will allow the robot to enter the larger connected network pipes which range from $50 \mathrm{~mm}-1250 \mathrm{~mm}$. By using a magnetic robot that does not rely on wall-pressing for traction the obstacles usually encountered in-pipe can be simplified and focus can be narrowed to just two cases. The robot has proven effective at 90-degree wall climbing cases where two magnetic forces are active at once and can generate tractive forces necessary to overcome the unwanted magnetic force. Further work will be done on the optimization of the circular magnetic array, such as testing N52 neodymium and reducing the rubber thickness. Smaller magnetic wheels are to be added to stabilize the rear of the chassis, and the electronic component stack will be integrated within the frame of the robot to allow it to drive on either side of the chassis.

\section{Acknowledgments}

This work was supported by National Grid Gas Transmission (NGGT) and its project partners Synthotech, Premtech, and Pipeline Integrity Engineers as part of Project GRAID: Gas Robotic Agile Inspection Device. ESPRC Grant Number: 1657711

\section{References}

1. Roslin, N. S., Anuar, A., Jalal, M. F. A., \& Sahari, K. S. M. (2012). A review: hybrid locomotion of in-pipe inspection robot. Procedia Engineering, 41, 1456-1462.

2. Mills, G. H., Jackson, A. E., \& Richardson, R. C. (2017). Advances in the Inspection of Unpiggable Pipelines. Robotics, 6(4), 36.

3. Lee, D., Park, J., Hyun, D., Yook, G. and Yang, H.-s. Novel Mechanisms and Simple Locomotion Strategies for an In-Pipe Robot that can Inspect Various Pipe Types. Mechanims and Machine Theory. 2012, 56, pp.52 - 68.

4. Yang, S.U., Kim, H.M., Suh, J.S., Choi, Y.S., Mun, H.M., Park, C.M., Moon, H. and Choi, H.R. Novel Robot Mechanism Capable of 3D Differential Driving Inside Pipelines. In: International Conference on Intelligent Robots and Systems, 14-18 Sept. 2014, Chicago, IL. IEEE, 2014, pp.1944-1949.

5. Kwon, Y.-S., lee, B., Whang, I.-C., Kim, W.-k. and Yi, B.-J. A Flat Pipeline Inspection Robot with Two Wheel Chains. In: International Conference on Robotics and Automation (ICRA), 9-13 May 2011, Shanghai. IEEE, 2011, pp.5141-5146Will 
6. Horodonica, M., Preumont, A., Burda, I. and Mignon, E. The Heli-Pipe Inspection Robots Architecture for Curved Pipes. In: International Conference of Manufacturing Systems. 2003.

7. Sato, K., Ohki, T. and Lim, H.-o. Development of In-Pipe Robot Capable of Coping with Various Diameters. In: 11th International Conference on Control, Automation and Systems, 26-29 Oct. 2011, Gyeonggi-do, Korea. IEEE, 2011, pp.1076-1081

8. Park, J., Hyun, D., Cho, W.-H., Kim, T.-H. and Yang, H.-S. Normal-Force Control for an In-Pipe Robot According to the Inclination of Pipelines. In: Transactions on Industrial Electronics, Dec. 2011. IEEE, 2011, pp.5304 5310.

9. Nishihara, T., Osuka, K. and Tamura, I. Development of a Simulation Model for Inner-Gas-Pipe Inspection Robot: SPRING. In: Society of Instrument and Control Engineers of Japan, 18-21 Aug, Taiwan 2010.

10. Dertien, E., Stramigioli, S. and Pulles, K. Development of an Inspection Robot for Small Diameter Gas Distribution Mains. In: International Conference on Robotics and Automation (ICRA), 9-13 May 2011, Shanghai, China. IEEE, 2011, pp.3447-3448.

11. Debenest, P., Guarnieri, M. and Hirose, S. PipeTron Series - Robots for Pipe Inspection. In: 3rd International Conference on Applied Robotics for the Power Industry, 14-16 Oct, Foz do Iguassu. IEEE, 2014, pp.1-6.

12. Schempf, H., Mutschler, E., Gavaert, A., Skoptsov, G. and Crowley, W. Visual and Nondestructive Evaluation Inspection of Live Gas Mains Using the Explorer Family of Pipe Robots. Journal of Field Robotics. 2010, 27(3), pp.217-249.

13. Kawaguchi, Y., Yoshida, I., Kikuta, T. and Yamada, Y. Internal Pipe Inspection Robot. In: International Conference on Robotics and Automation, 21-27 May 1995, Nagoya. IEEE, 1995, pp.857-862.

14. Tache, F., Fischer, W., Caprari, G. and Siegwart, R. MagneBike: A Magnetic Wheeled Robot with High Mobility for Inspecting Complex-Shaped Structures. Journal of Field Robotics. 2009, 26(5), pp.453-476.

15. Synthotech Limited. [Online]. 2018. [Accessed: 16/04/2018.] Available from: http://www.synthotech.com/

16. National Grid 2018. Project GRAID. [Online]. 2018. [Accessed: 16/04/2018.]. Available from: http://projectgraid.com/

17. Boonyaprapasorn, A., Maneewarn, T., \& Thung-Od, K. (2014, October). A prototype of inspection robot for water wall tubes in boiler. In Applied Robotics for the Power Industry (CARPI), 2014 3rd International Conference on (pp. 1-6). IEEE.

18. Tavakoli, M., Viegas, C., Marques, L., Pires, J. N., \& De Almeida, A. T. (2013). Omniclimbers: Omni-directional magnetic wheeled climbing robots for inspection of ferromagnetic structures. Robotics and Autonomous Systems, 61(9), 997-1007. 